Chapter 4

\title{
Marine Biodiversity and Chemodiversity - The Treasure Troves of the Future
}

\author{
Stéphane La Barre \\ Additional information is available at the end of the chapter \\ http://dx.doi.org/10.5772/57394
}

\section{Introduction}

Chemodiversity usually refers to small molecules that have a signaling (offensive or defensive) function, sometimes protective. This leaves aside larger molecules that are purely structural and those that participate in essential metabolic functions, and make up the bulk of the organic body mass of living organisms.

From cyanobacteria and bacteria to the largest metazoans, chemistry is the preferred mode of aquatic communication, thanks to the extraordinary solvation properties of water. Bacteria create biofilms inside which they communicate using their own chemical repertoire before colonizing new media, substrates or organisms. Microalgae form blooms which are maintained by releasing semiochemicals for cell-cell recognition. Fish rely on their extraordinary sense of smell to hunt or to migrate to some specific breeding spot. The extraordinary biodiversity of coral reefs is maintained by a highly complex chemical network of toxins and pheromones, some soluble, some dispersed with a mucus carrier or surface-coated. But not only: the amazing colors used for warning or for camouflage, the bioluminescence used in the dark correspond to very sophisticated assemblages of pigments, small metabolites or proteins, each organism having its own strategy to be visually recognized or to blend into the background.

Humans have only recently been aware of the extraordinary potential marine molecules for the design of new drugs, cosmetics and nutraceutics. Well over 20000 natural molecules have been studied so far, and several have responded to the need for novel anticancer, antibiotic, anti-inflammatory or anti-pain agents etc. The necessity to preserve this exceptional resource, however, has only manifested itself in the delineation of protected areas and in the implementation of codes of good practices regarding non-destructive boating and durable sampling protocols. 
Over the last three decades, warning messages have been sent to the community about the destructive consequences worldwide economic development will have on biodiversity, both terrestrial and marine, during the 21st century. Direct impacts are caused by overexploitation and mismanagement of natural resources and improper recycling and disposal of waste products. Indirect impacts are caused by the accelerating volatilization of greenhouse molecules and their accumulation in the atmosphere where they may undergo undesirable speciation. Restitution of sulfur emissions to land may cause acidic rains and transfer of carboncontaining emissions to seawater increases its acidity, both leading to biodiversity destructive scenarios. Not to mention the release of man-made (synthetic) molecules, some of which like CFCs destroy the anti-UV ozone shield, others like PCBs accumulating along food chains and eventually killing top consumers. Synthetic molecules may respond to specific needs and criteria, but they will never replace natural molecules, in the same way as genetically transformed organisms will never replace wildlife diversity. Moreover, freak biological or chemical species should be eliminated safely once the purpose for which they were created has been fulfilled.

To-date, very little is said or written on the fate of natural chemodiversity within the context of local or general biodiversity collapse, both terrestrial and marine. After a brief historical account of the intricate connections between chemodiversity and biodiversity since life appeared on our planet, this chapter attempts to demonstrate that natural molecular diversity is a treasure to preserve for future generations, using a series of marine examples.

\section{Early chemodiversity was non-biotic}

\subsection{From single elements to simple organic molecules in a mineral world}

Chemistry is as ancient as the observable universe, ca. 13.7 billion years old according to present estimates. The simplest elements of the periodic table (namely, hydrogen, helium and traces of lithium) were formed as an immediate consequence of the Big Bang.

The nuclear chemistry of the galaxies and stars which developed during the following few billion years is the chemistry of very high energies, yielding a small and finite number of chemical species, some very short-lived. Carbon, oxygen and nitrogen came to existence. The data on star-forming elements is of spectroscopic nature. When decomposed and analyzed, the light emitted by these objects reveals the presence of spectral lines at set wavelengths, and their association forms fingerprints typical of individual elements when heated to incandescence. The solar system was formed about 4.6 billion years ago by the accretion of simple molecular substances from a giant cloud at the center of which most the concentrating matter formed the sun, the rest forming a disc from which primary planets, then satellites planets emerged under various scenarios.

Under very hot temperatures and no atmosphere, the molecular chemistry associated with proto-planets is dominated by inorganic entities, with very limited diversity in carbon chemistry. Carbon dioxide and water probably existed as soon as oxygen, a stellar product, 
had been available to combine with hydrogen, yet early planet Earth was most likely very hot and dry, precluding life and any of the complex chemistries it produces. This characterized the Hadean, the first geologic eon (4.6 to 4.0 billion years until conflicting evidences such as the presence of high $\mathrm{d}^{18} \mathrm{O}$ (oxygen isotopic ratios) in zircons tentatively dated as early as 4.4 billion years led to a reassessment of the transition between the "molten" planet (and formation of the moon) and the "solid" planet with a solid crust, a low temperature, and an atmosphere of sorts, and the possible presence of liquid water. As well as this "cool early earth" hypothesis [1], a partial explanation about the formation of oceans is that asteroids and perhaps comets carrying huge amounts of ice collided with our planet, adding to the putative de novo condensation into water during the degassing of rocks of the cooling planet. In the primitive atmosphere, heavier carbon dioxide became partly quenched by dissolving in oceanic waters, and partly trapped under plate tectonic movements.

\subsection{The chemical origin of life on earth - Chemodiversity goes organic}

Any life form necessitates the capacity to harvest energy for its own benefit, to manage chemical reactions within molecular boundaries that define it as an entity distinct from the environment, i.e. self vs. non-self, and to have the potential to replicate itself.

Irrespective of when the right conditions were first met for life to emerge, a small set of small organic molecules (reactants) is a prerequisite for the abiotic generation of life-essential molecules, in the presence of water and of some catalytic trigger, and later of thermodynamically favorable conditions for polymers to be built.

Different approaches have attempted to address the question of the chemical origin of life, leading to apparently conflicting conclusions: the "prebiotic soup theory" and the "pioneer organism theory", fueling heated debate among specialists, and also reflecting the uncertainties that still remain on the greatest mystery in science. The following paragraph attempts to follow the most generally accepted views and the most plausible scenario.

\subsubsection{The Miller-Urey laboratory experiments}

The prebiotic soup theory stems from the early experiments of Stanly Miller and Harold Urey [2] which led to the condensation within days of a suite of five amino acids by exposing a sterile mixture of methane $\left(\mathrm{CH}_{4}\right)$, ammonia $\left(\mathrm{NH}_{3}\right)$, carbon monoxide $(\mathrm{CO})$ and hydrogen $\left(\mathrm{H}_{2}\right)$ - i.e. the "primitive gases", to a constant flux of hot water $\left(\mathrm{H}_{2} \mathrm{O}\right)$, while discharging electric sparks (to simulate thunder) into the gas mixture. New species such as hydrogen cyanide (HCN), formaldehyde ( $\mathrm{HCHO}$ ) and reactive intermediates (acetylene, cyanoacetylene etc.) are formed in one-step, while amino acids and other biomolecules are formed from these reactants under reducing conditions (Fig. 1), i.e. the Strecker synthesis.

Some 50 years later, Bada and collaborators (in [3]) reanalyzed archived samples of Stanly Miller's experiments in which the gaseous mixture included hydrogen sulphide $\left(\mathrm{H}_{2} \mathrm{~S}\right)$, methane $\left(\mathrm{CH}_{4}\right)$, ammonia $\left(\mathrm{NH}_{3}\right)$, and carbon dioxide $\left(\mathrm{CO}_{2}\right)$. In the case of this experiment (unreported by Miller at the time), no less than 27 compounds, including the 20 "regular" amino acids and 3 new ones were found. Specifically, the presence of hydrogen sulphide and carbon 
dioxide as reactants - originally proposed to simulate the influence of volcanic emissions - had enriched the diversity of the reaction products with seven several sulphur-containing compounds, including methionine and cysteine), and four amines as well.

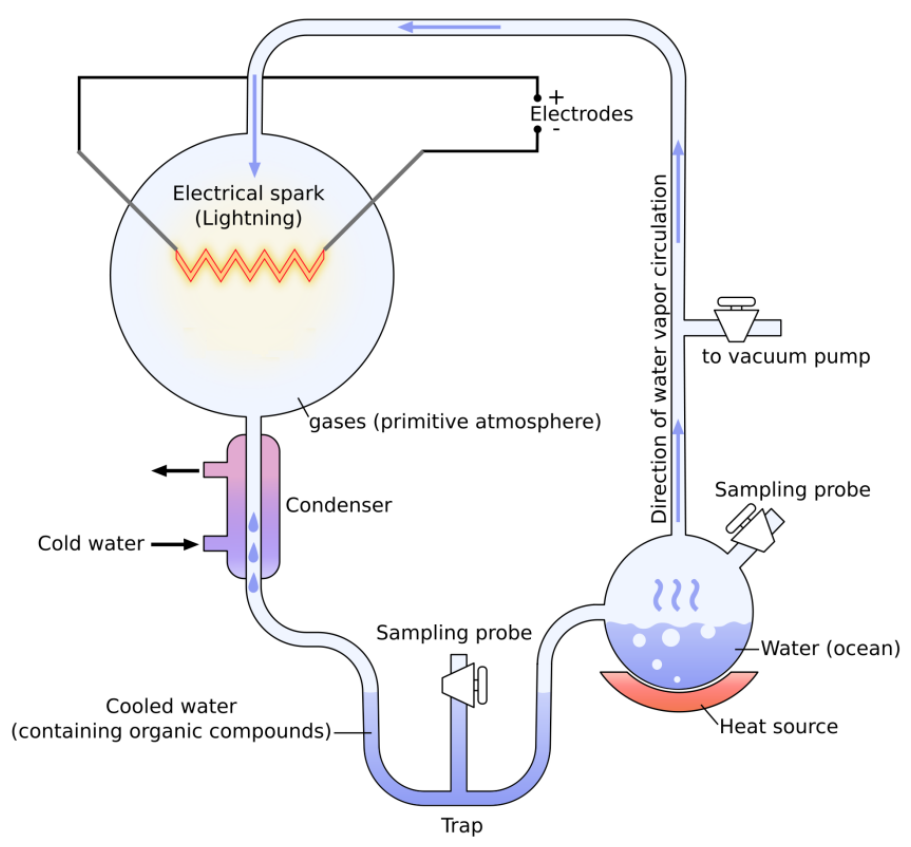

Figure 1. The apparatus used by Miller and Urey in their 1953 simulated early-earth experiment on the chemical origins of life. In the original experiment, the gases of the primitive atmosphere were: $\mathrm{NH}_{3}, \mathrm{CH}_{4}, \mathrm{H}_{2}$ and water vapor. Other experiments were carried out under non-reducing conditions (using $\mathrm{CO}_{2}, \mathrm{~N}_{2}$ and water) and UV exposure led to poor yields. In experiments simulating volcanic environments, Miller and Urey used $\mathrm{H}_{2} \mathrm{~S}, \mathrm{CH}_{4}, \mathrm{NH}_{3}$ and $\mathrm{CO}_{2}$ (see text). (Graphics by Yassine Mrabet in http://en.wikipedia.org/wiki/Miller-urey_experiment)

\subsubsection{Other "prebiotic" laboratory experiments}

Oró and collaborators [4] using similar experimental setups, established that adenine and other nucleic bases, as well as several amino acids, could be formed from $\mathrm{HCN}$ and $\mathrm{NH}_{3}$ in water, under reducing conditions.

In addition, simple sugars can be formed under putative prebiotic conditions [5], an essential feature for the emergence of nucleosides [6] and RNA (qv. the RNA world). The Butlerow reaction, i.e. the synthesis of a complex mixture of sugars (including ribose and arabinose) from formaldehyde by the action of catalysts such as calcium hydroxide, has been known since the 19th century under laboratory conditions. 


\subsubsection{Transposing to "real" prebiotic conditions}

In order to transpose from lab experiments to real life situation, several criteria must be met in order to initiate amino acid synthesis: the reactants must be concentrated enough under an aqueous environment under favorable temperature and $\mathrm{pH}$ conditions.

The primitive soup theory relies on a stochastic occurrence of optimal conditions for the emergence of organic life. Polymerization necessitates suitable absorbing substrates and catalysts such as metal cations and imidazole derivatives and several others, to proceed in laboratory conditions. Charged submicronic montmorillonite clay particles would have helped activated monomers to selectively concentrate (electrostatic bonds) and induce covalent polymerization. Shallow lagoons in tidal zones may theoretically provide basic chemistry. Evaporation induces concentration and promotes the formation of eutectic complexes.

Contenders of the "primitive soup" scenario advocate that life may have arisen through chemoautotrophic processes occurring in oceanic depths in the vicinity of hydrothermal settings which would provide all necessary starting conditions [7]. In this "pioneer metabolism" scenario, the generation of homochiral metalloenzymes of extant organisms from inorganic transition metal precipitates (by chelation of alpha-hydroxyl and alpha-amino acids ligands) follows a stepwise evolution by autocatalytic feedback. This "hot volcanic" prebiotic chemistry is often opposed to the "cool oceanic" chemistry that typifies the primitive soup scenario.

It appears that the composition of the primitive atmosphere is not what it was thought to be at the time of Miller's experiments, certainly not as reducing, and the conditions afforded in the hydrothermal vent environments appear more amenable to prebiotic chemistry. Oligomers could have then been formed at liquid-solid interfaces [8].

However, prebiotic chemistry under early atmospheric conditions has opened the issue towards exobiology. Scientists of repute now speculate on an extraterrestrial origin of life, or at least in the alien seeding of life essential molecular building blocks on planet earth. But this is another story.

\subsubsection{Before and during the RNA world}

The recent finding that ribonucleic acids (RNAs) can perform a variety of hitherto unsuspected structural and metabolic functions in cells has given credit to Walter Gilbert's prediction [9] that an all-RNA world had preceded the nucleic acid-protein world as we know it today. The fact that RNA takes evolutionary precedence on DNA (itself regarded by some as a modified RNA better suited for the conservation of genetic information), or the fact that RNAs as a catalysts (ribozymes) take precedence on enzyme catalysts, stimulate intense experimental interest.

Nucleotide monomers can theoretically be surface-assembled as oligomers [10]. The longest strands serving as templates, direct synthesis of a complementary strand starting from monomers or short oligomers, and double-stranded RNA molecules can accumulate. Disso- 
ciation of strands, one of which endowed with RNA polymerase activity, would lead to successive replication processes. Finally, the RNA world would have emerged from a mixture of activated nucleotides. However, the precise molecular mechanisms which initiated RNA oligomers in the first place, remain obscure. The monomers (nucleotides) must be activated and homochiral, before they can be assembled as strands on an absorbing mineral surface (e.g. montmorillonite clay) acting as a catalyst.

Interestingly, other nucleotide-like structures that may be formed under prebiotic conditions can reasonably qualify as RNA functional analogues and have been termed "alternative genetic systems" [11].

The existence in prebiotic conditions of AMP-derived cofactors that have acquired amino acidlike properties through post-translational modifications is possible, since these cofactors participate at all levels in the metabolism of extant life forms. As Maurel and Haenni [12] put it," Coenzymes would be vestiges of catalytic nucleic enzymes that preceded ribosomal protein synthesis, and tRNAs can be viewed as large coenzymes participating in the transfer of amino acids."

Ligand-substrate recognition must have followed from the selection of aptamers (molecules that bind specifically to one target molecule) from a pool of oligonucleic acids or peptides. Artificial selection of adenine-dependent ribozyme aptamers [13 ] indicates that the use of small exogenous cofactors (adenine) by ribozymes could have actively contributed to the expansion of the catalytic and metabolic repertoires of RNA species in the prebiotic RNA world. Modern catalytic equivalents of ribozymes are metalloenzymes, and adenine fills the same cofactor function as histidine.

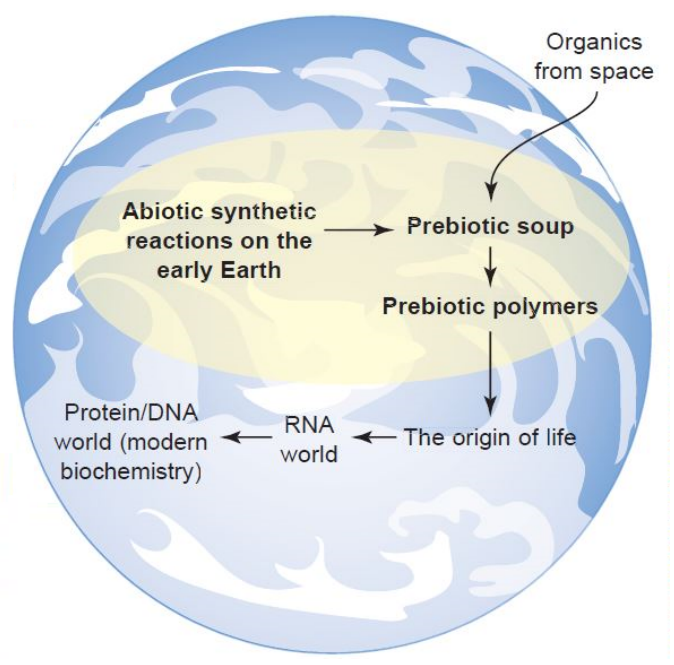

Figure 2. A depiction of the step-by step scenario involved in the origin of life on earth, attempting to integrate the "metabolist" scenario into the "primitive soup" scheme (from: Bada and Lazcano, [14]) 
Indeed, the RNA world was probably much more chemodiverse than sometimes described (a uniform soup), notwithstanding the fact that, as said before, tidal lagoons with the presence of catalytic elements such as clay submicronic particles, or the vicinity of hydrothermal vents would greatly favor polymerization and functional gains of prebiotic organic molecules.

\section{Extra-terrestrial chemistry going organic}

Recent investigations [15] have led to the identification of many $(>100)$ prebiotic molecules (HCN, HCHO, glycoaldehyde etc.) in the composition of interstellar clouds and in the gasphase chemical evolution in the atmospheres of various planets (i.e. corresponding to the first step of the Miller-Urey experiment). If the radicalar processes leading to the formation of these intermediate species are simple compared to the sophisticated biochemistry involved in terrestrial chemodiversity, they remain largely unknown and should help us better understand prebiotic chemistry. Different types of molecular processes are thought to be involved, including radiative association and recombination, surface-induced processes, photon or particle induced ionization, ion-molecule reactions, photon or particle induced dissociation and radical-molecule interactions.

Moreover, amino acids are known to occur extra-terrestrially since the 1970 discovery in the Murchison meteorite, of over 70 common and exogenous species under non-racemic proportions, some under relative abundances similar to those found with the original 1953 MillerUrey experiment.

This was made possible through the activation of a multiple-component system [16].

This raises the problem of chirality, since on our planet, natural amino acids are all left-handed (L-amino acids). It is speculated that the prevalence of the L- form may have been influenced by polarized radiations from outer space [17]. In the laboratory, crystallization experiments in a racemic mixture always follow the same enantiomeric form as that of the initiating nucleus, further amplifying the "preference" for the L-form. Homochirality is an essential feature of biopolymers, for which correct folding must be required for proper function [18].

\subsection{From proto-cells to living entities}

\subsubsection{Self-assembling vesicles}

Self-organization of amphiphilic molecules (i.e. long chain fatty acids) into bilayers or into vesicles can be readily observed in laboratory conditions. In live cells, membranes provide the most elementary delineation of the self from the non self, i.e. it defines the basic identity of an individual from its environment. In non-living systems, vesicles can selectively separate solutes according to their affinities (polar or apolar) and even to their chiral properties [19].

\subsubsection{The amphiphilic double layer}

In particular, the double layer which separates the vesicular contents from the outside can selectively extract lipophilic substances from the environment and arrange them around the inner (hydrophobic) tails. Experimentally, this feature promotes concentration of e.g. apolar 
amino acids and their non-enzymatic condensation into dipeptides or small oligopeptides within the lipid bilayer. In addition, vectorial properties in phospholipidic vesicles are thought to influence the inner $\mathrm{pH}$ (making it lower than outer $\mathrm{pH}$ ) in small vesicles, due to differences in the behavior of water molecules.

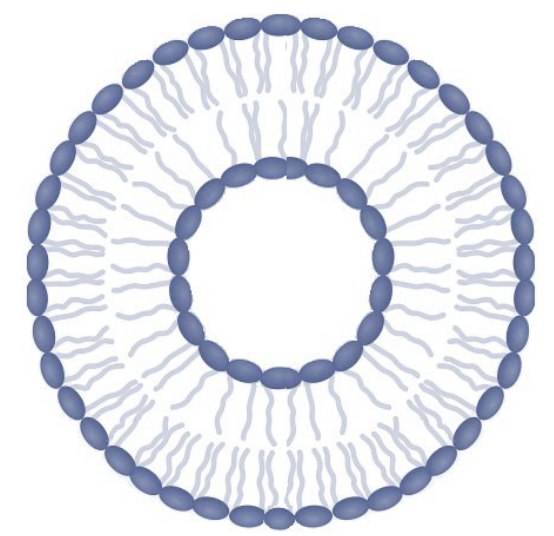

Figure 3. A phospholipid vesicle, with a double-layered membrane. Each layer includes a hydrophilic head (dark knobs) and a hydrophobic tail made up of long lipid chains. The aqueous environment inside the vesicle isolates entrapped metabolites, biopolymers and catalysts, the whole structure acting as a reaction vessel.

\subsubsection{Vesicles as proto-cells}

The total synthesis of a simple cell is possibly the ultimate challenge in synthetic biology. It is experimentally possible to entrap nucleic acids inside self - forming phospholipid vesicles, acting as reaction vessels for e.g. polymerase chain reaction, enzymatic RNA replication and even protein synthesis, as demonstrated by Oberholzer and collaborators (reviewed in [19]). The molecular pump scenario [20], in which peptides were initially synthesized through a molecular engine could also have taken place inside such structures. In nature, the inclusion of clay microparticles acting as catalysts is highly probable. Thus, phospholipid vesicles can be regarded as useful experimental models of proto-cells [21], but many questions still need addressing before considering them as early precursors of living cells.

Protobiology uses a bottom up approach when designing complex and functional protocells from simple molecules and organic catalysts.

Synthetic biology uses a top-down approach to identify the minimal components of living cells that will qualify, in other words to rediscover the basic cell before it underwent the complexities of Darwinian evolution. Thus protocells are of exciting biotechnological interest in the quest to optimize chemotherapies (cellular target recognition, kinetics of drug delivery).

Prebiotic chemistry is certainly more complex than previously thought, and chemodiversity has undergone periods of expansion, especially through stochastic combinations during the so-called RNA world, and during the development of proto-cells. 
Chemodiversity as we know it today is mostly organic and is the result of complex biochemical processes within living organisms that must constantly adapt to changing environments and compete for their survival.

\section{Chemodiversity explodes with the emergence of life}

\section{1. "Cool" carbon chemistry}

Our chemodiversity is usually classified as mineral or organic. Exploitable minerals are solidified in the earth crust or deposited as sediments or conglomerates - geochemical cycles connect the molten inner core of the planet with the biosphere through the displacement of continental plates and volcanic activity. Organic chemodiversity is concentrated in the biosphere, as the result of the present and past metabolic processes of marine and terrestrial organisms. Chemically, organic molecules are carbon atoms and scaffolds of connected carbon atoms, linked to hydrogen and covalently to heteroatoms e.g. oxygen, nitrogen, atoms but also to sulfur, phosphorus and halogens or metal complexes (coordination chemistry) to form functional groups. Larger organic molecules often adopt three-dimensional architectures that confer them with the exquisite specificity required for recognition and binding to a defined molecular target. Organic natural products are by far the largest contributors to marine and terrestrial chemodiversity, and also the most labile in the face of anthropic and natural influences that affect the stability of our ecosystems.

\section{2. "Cool" oxygen biochemistry}

The chemistry of our oceans has evolved considerably [22], especially with the gradual production of oxygen, which initially oxidized mantle rocks and dissolved reduced metals such as iron then tended to precipitate, as evidenced by red strata in ancient sediment beds. Free oxygen then started to rise in atmospheric concentrations, some of which was split by cosmic radiations to form the protective ozone layer. Respiration became possible and some life forms moved out of the oceans, and both aquatic and terrestrial species diversification started, and with it, organic chemodiversity. It should be emphasized that combustion occurs at oxygen levels only above $15 \%$ and organic matter would spontaneously burn at $25 \%$ concentrations - which explains that life is possible only if the present level (21\%) is maintained around $20 \%$ and if genotoxic radiations can be efficiently filtered out. Oxygen is also toxic at the cellular level, if the production of (or exposure to) reactive oxygen species (ROS) or oxygen radicals cannot be counterbalanced by antioxidants or if the resulting damages cannot be repaired. When involved in regular intracellular signaling, ROS can be involved in the activation of defense metabolic pathways, and contribute to the enrichment of the metabolic repertoire of planktonic and benthic organisms.

\subsection{Biochemistry operates under very specific conditions}

With the exception of very few extremophiles that can live under elevated temperatures (above $80^{\circ} \mathrm{C}$ ) and hyper acidic environments $\mathrm{pH}<2$ ), most microorganisms are not tolerant to 
conditions found outside the biosphere. The biosphere represents a very narrow region less than one thousand times thinner than the globe's diameter, and comparable conditions have not been found on any other planet so far. This does not mean that dormant bacterial spores could not withstand intersidereal journeys - lichens have successfully resisted prolonged exposures in outer space during space craft experimentations. High biodiversity environments are usually found in warm, stable environments where nutrient cycling is efficient (without accumulation of wastes) - this is at least true for tropical rainforests and shallow water coral reefs.

\section{Biodiversity explosions and mass extinctions (from [26])}

"Any lasting change in the biogeochemistry of any of the three components (atmosphere, seawater and land) will disrupt the interfacial equilibrium that supports the many thousands of life forms that interact constantly within an ecosystem. This has happened several times in the history of our planet since it became life-supporting. Changes in soil mineral strata indicate the occurrence of biodiversity-modifying events such as occupation by seas or the occurrence of an ice-age. Discrete organic layers may indicate the presence of a tropical rainforest or of a dry land savannah. Datable fossil evidence within these strata, together with paleontological reconstructions, point out the floristic and faunistic peculiarities of the times. Core drills in ice provide datable evidence of biogeoclimatic episodes within the last few millennia, while core drills in massive scleractinian corals give accurate calendar-like records of recurrent or of accidental climatic events affecting their biotope.

Speciation usually goes along with occupation of new territories and new habitats, the first colonizers having acquired the necessary adaptations to cope with evolving external demands - the Cambrian explosion (545 million years ago) being the most dramatic example of such adaptive diversification at all scales.

Along with this, evidences of accidental episodes of massive species extinctions are witnessed by the sudden "disappearance" of terrestrial and of marine life, that are attributable to tectonic, telluric or meteoritic impacts and to their profound and lasting climatologic and geochemical consequences. The most significant mass extinction is undoubtedly the Permian-Triassic Great Dying [23] where a 96\% loss of all non-microbial marine life occurred within ten million years. The precise causes of mass extinction events may be in connection with continuous tectonic movements with their telluric and volcanic outbreaks and their climatic consequences, to collisions with meteoritic bodies, and to a lesser extent to the appearance of dominant predators, parasites or microbial diseases, or to combinations thereof. Common with many extinction events, however, is the massive release of greenhouse and of toxic gases (carbon dioxide, methane, hydrogen sulfide etc.). The water solubility of $\mathrm{CO}_{2}$ being nearly 30 times that of oxygen, water acidification occurs that impacts preferentially all calcifying organisms with low metabolic rates and weak respiratory systems: most coral genera died out during the Great Dying, along with calcareous sponges, calcifying algae, echinoderms, bryozoans etc. [24].

Interestingly, profound taxonomic changes in all major phyla seem to follow extinction events, resulting in a better adapted biodiversity. Nothing is known, however, on the consequence of 
such changes in microbial life or on the putative role microbial associates had on the reinvention (understand: adaptive evolution) of new species. As Falkowski [25] puts it, animals and plants are merely new incarnations of ancient metabolic processes, but the ultimate key to biodiversity may be held by bacteria ferrying the set of core genes that are necessary for life to express itself ".

\section{Marine biodiversity and chemodiversity today}

Water is an extraordinary medium and, in many respects, mysterious. Its outstanding solvation properties are due to the capacity of the water dipoles $(\mathrm{H}-\mathrm{OH})$ to dissociate, reorientate and accommodate salts and polar compounds in a monophasic system [27]. Salts are essential to maintain osmotic balance and membrane polarization. Hydration is essential for the catalytic properties of enzymes. Semiotic (communication) substances are usually released as pheromones (intra-specific signaling) or allomones (against defense or predation). In addition, apolar (long chains or cyclic) molecules can be associated with mucus and dispersed. Some chemical cues are surface-adsorbed on dead substratum and serve as transgeneration signaling, e.g. for the settlement of larvae near adult colonies. Fish have extraordinarily sensitive and selective receptors that allow them to detect specific chemical signatures that influence their behavior.

Water participates in all life-essential molecular processes, from the most basic (e.g. Miller's experiment) to the most complex enzymatic processes, and it has allowed virtually all types of interatomic and molecular interactions that have resulted in the highly complex and diverse chemical diversity observed in our oceans today.

\subsection{Minerals and geochemical cycles}

The mineral world is massively involved in global geochemical processes, and in the composition of solutes in the oceans, as salts and inorganic carbon sources. The cycling of elements is both tectonic and biogenic. Metals are essential catalysts of many organic reactions, and salts are actively involved in osmotic balance and membrane polarization. The availability of heteroatoms ( $\mathrm{P}, \mathrm{S}$, halogens in particular) is (and has been originally) fundamental in the evolution of natural organic chemistry, because of their involvement in life-essential processes.

\subsection{Prokaryotes and prokaryote chemistry}

"Creative" chemistry is the work of living creatures, and Eubacteria and Archaea are probably the best organic chemists ever. Microbes are the drivers of global biogeochemical processes, and their genes have survived the great extinction events [28]. They have set the stage for other organisms to adapt and evolve. The capacity for pioneer organisms to modify their environment for the benefit of other life forms is termed metabiosis, and it has been largely documented in soil biology [29], but somewhat overlooked in aquatic ecosystems. They can occupy every niche of the biosphere, utilize different carbon and/or energy sources available in order to generate ATP, the energy currency of all biochemical processes. 
A distinction is made between chemotrophs and phototrophs as primary producers of organic molecules.

\subsubsection{Chemotrophs}

Chemotrophic Archaea and Bacteria that live in oxygen-depleted environments (e.g. in the vicinity of hydrothermal vents) derive their energy from the oxidation of reduced inorganic compounds and use (i) carbon dioxide as sole carbon source or (ii) lipids, sugars or proteins to form their own organic compounds. Many scientists believe that life originated in the oceans, in the absence of light and in the vicinity of underwater tectonic/volcanic activity, under similar conditions to post-Hadean times.

Archaea are recognized among the most abundant living entities in the oceans, participating in major biocheochemical cycles, such as the Methanogens that produce most the atmospheric methane greenhouse gas. Halophilic Archaea thrive in hyper saline environments like in the Dead Sea, sometimes in hyper alkaline conditions ( $\mathrm{pH}$ around 12). On the other hand, extremophilic Archaea can be found in hot sulfur springs under $\mathrm{pHs}$ as low as 2. They are either anaerobic and reduce hydrogen sulfide to sulfur, or aerobic and oxidize sulfur to sulfuric acid. Thermophilic $\left(50-70^{\circ} \mathrm{C}\right)$ and hyperthermophilic $\left(70\right.$ to $\left.118^{\circ} \mathrm{C}\right)$ Archaea produce thermostable enzymes of major biotechnological importance, e.g. in DNA amplification using polymerase chain reaction (PCR), in food industry, etc.

Chemotrophic Eubacteria include chemoautotrophs which utilize inorganic sources and environmental $\mathrm{CO}_{2}$, (like nitrogen-fixing soil bacteria, iron and manganese-oxidizing hydrothermal bacteria) and chemoheterotrophs which degrade existing organic substrates. Many bacteria (Gram-positive Actinomycetes, Bacillus, Gram-negative gamma-Proteobacteria) are chemotrophs, and produce a wide array of bioactive compounds, many of which have strong antibiotic potential (examples are given in [30] vol.1, chapter 7). Interestingly, hydrothermal vents are crowded with invertebrates (mollusks, crustaceans and worms) that cultivate chemotrophic bacteria that provide them with organic nutrients.

\subsubsection{Phototrophs}

Phototrophs harvest solar photons as the energy source to carry out their cellular energy processes.

Most phototrophs carry out photosynthesis, a process by which carbon dioxide is converted into organic material, used structurally (generally as polymers or supramolecular complexes) or functionally for the regulation of cellular processes, or stored as reserves.

A trans-membrane electrochemical gradient is created, which is utilized by ATP synthase, to create ATP (adenosine triphosphate), the key molecule that fuels biochemical processes in the cell, from the oxidation of glucose. Oxygen is generated in the process. Archaea may fix carbon and some use light as energy source to create metabolic energy but none is capable is capable of photosynthesis with the use of complex electron- transfer chains. Halophilic archaea use bacteriorhodopsin instead - a pigment that activates a transmembrane proton pump. 
Photosynthesis began as an anoxygenic process that later evolved to produce oxygen, thus allowing the evolution of aerobic metabolism. Photoautotrophs include most producers of primary biomass, from cyanobacteria to algae, plants and are involved as photosymbionts in vegetal (e.g. lichen) and animal (e.g. coral) biological photosystems. They are the base of simple or highly complex marine and terrestrial food chains.

\subsubsection{Heterotrophs - the good, the bad and the ugly}

Many bacteria derive their energy from the breakdown of organic substances produced by living or dead multicellular organisms. They are instrumental in the recycling of dead organic matter, and many strains live as commensals or symbionts of most eukaryotes, to help the host's digestion with nutrient assimilation or with the energy-yielding catabolism of proteins, carbohydrates and lipids into small, usually water-soluble monomers. A highly diverse microflora is generally associated with good health at the single host's level as well as whole ecosystems. Pathogens are maintained but their numbers are regulated by complex peerinduced antibiotic and bacteriostatic pressure. In corals, metabolic efforts to adapt or compensate lasting stress (temperature, salinity, hypoxia sometimes in combination) will affect the host's resistance, favor the dominance of opportunistic and aggressive strains and dramatically reduce microbial diversity, leading to a range of necrotic diseases. In humans, obesity and consequential diabetes and cardiovascular diseases strongly correlate with poor feeding habits and deficient gut microflora [31].

Some strains are downright lethal after acquiring multi-resistance to existing antibiotics, and if provided with conditions that trigger their inherent but normally unexpressed pathogenicity. Some exciting scientific advances have been made since the discovery of quorum sensing in bacteria, the detailed study of microbial biofilms, and in the understanding of lethal epidemics (e.g. caused by Vibrio species). However, by and large, metagenomic studies have clearly shown that eukaryotic and prokaryotic biodiversity (and hence chemodiversity) are strongly correlated.

\subsection{Eukaryotes and Eukaryote-associated chemistry}

Cyanobacteria are thought to have enriched the primitive atmosphere in oxygen, promoting biodiversity through repeated endosymbioses, i.e. successive fusion-acquisition processes that led to the complex metabolic machineries of higher plants (origin of plastid organelles) while ancestral bacteria became incorporated as mitochondria in most eukaryotes. Other organelles are suspected to have generated from similar mechanisms. Many eukaryotes have successfully colonized non-aquatic environments.

This increase in biodiversity has itself led to competition for existing resources: food, space, access to light etc.

In non-motile organisms (algae, halophytes and some benthic invertebrates) in which escape responses are not possible, species-specific assortments of physical protections and/or chemical defenses have evolved to discourage predators. Their remains are collectively termed biominerals. Some growth forms (encrusting, massive, cryptic) may also 
resist whole-organism or whole-colony predation, or restrict their access.

Algae are generally non-toxic, but may contain antifeedants such as organohalogens or polyphenols that are distasteful to fish [32].

Not all sessile, slow-moving and sedentary organisms enjoy an efficient physical protection. Fleshy invertebrate species and those that must be exposed to sunlight to perform photosynthesis may produce toxic or distasteful substances to discourage feeding by fish, crustaceans or other carnivores. Alcyonarian octocorals shelter photosymbiotic dinoflagellates, and are able to produce complex bouquets of cyclic terpenoids that are dissuasive against predators and efficient against space competitors. Sponges shelter biodiverse archaean, eubacterial and cyanobacterial communities and produce an amazing variety of metabolites (many are nitrogen containing and halogenated) as a functional consortium. Bryozoans shelter bacteria that produce highly complex and toxic molecules (e.g. bryostatins). Ascidians can produce highly bioactive molecules, such as the depsipeptidic didemnins, in association with prochloron, i.e. cyanobacteria which they cultivate in their tissues. The purpose here is not to list the thousands of amazing molecules that are produced by benthic marine invertebrates, to which we must add the recently discovered highly diverse repertoire of planktonic organisms: [31] and its updated version [33] in press) and [34] are probably the most recent and comprehensive texts on the subject.

Evolutionary reflections of chemical defenses in marine symbiotic systems have been recently proposed by [35]. Symbiotic and photosymbiotic systems that associate an invertebrate or algal host and its specific microbial consortium are common in tropical shallow water reefs, which concentrate at least one third of the total marine biodiversity and certainly the largest proportion of all known "secondary" metabolites [26]. To these must be added marine fungi and actinobacteria that live in marine sediments.

A generally overlooked component of marine chemodiversity is mucus, sometimes constantly and abundantly produced by epithelial goblet cells of cnidarians, and also found in other sessile invertebrates, and also in fish. No two muci are alike. In fish, it helps protecting scales from unwanted fouling by encrusters or parasites, as well as having intrinsic antibacterial and antiviral properties. Coral mucus has species-specific composition and plays a role equivalent to the organ-forming mesoderm of triploblastic organisms, in managing a highly biodiverse microbial fauna that recycles carbon, nitrogen and sulfur and provides an energy complement to symbiotic photosynthesis. To the coral associated bacteria mucus plays the role of a biofilm in which complex antibiotic interactions are at play to maintain microbial diversity.

Behavioral adaptations like "advertising" (aposematic) colors and body shapes make use of colored metabolites borrowed from the prey organisms and concentrated into superficial diverticula. Specialist predators like shell-less mollusks like dorid nudibranchs are beautifully colored and highly toxic. Several aeolid nudibranchs on the other hand use camouflage for similar reasons. 


\section{Uses of marine natural products}

Marine biogenic molecules are used in a large variety of applications, industrial, medical, pharmaceutical, cosmetological, as food or associated to food, decoration...

\subsection{Housing and transportation matters}

These include the use of fossilized or detritic materials for construction and as chemical energy sources.

\subsubsection{Construction materials}

Biogenic sand and rocks (e.g. sandstone) are used to create building, roads and associated structures. Urban architecture is mostly made of concrete structures, i.e. cemented biogenic or mineral sand and crushed rocks or debris.

\subsubsection{Fossil fuels and biofuels}

Fossil fuels are consumed at the rate of 88 million barrels a day, in heat engines, to produce electricity, domestic heating, and in petrochemical chemistry (plastics, clothing, lubricants, polymers). Fossil fuels are non-renewable energy, since the production rate is about onemillion times slower the consumption rate.

\subsection{Health and well-being}

This includes the use of biomineral or bioorganic materials in surgery, of cosmetics and skin care substances, and most of all of anticancer, antiviral, anti-pain, antibiotic, anti-inflammatory, as well as insecticidal, anti-malarial or anthelminthic molecules, not to mention other domains of growing interest, such as Alzheimer's disease and schizophrenia.

The exploitation of marine natural products in folk medicine is very limited and concerns a few organisms that did not necessitate special underwater investigations, nor entail storage and conservation problems - of paramount importance since desiccation, oxidation and fermentation occur so rapidly. Exceptions are the occasional use of specific algae as insecticides and for ringworm treatment, or the ceremonial use of toxic or venomous organisms. Fixed and fleshy organisms were generally distasteful or toxic, sparing them for being considered as remedies. Corals, sponges and a few others have no nutritional value and represent the largest biomass of high-diversity systems in tropical zones.

It is only since just after WW2 that marine organisms emerged as a complementary source to traditional plant remedies, thanks to the co-occurrence of several factors, among which (i) the recent discovery of fungal antibiotics and the use of bacterial enzymes in bioprocesses, (ii) the development of adequate screening procedures, (iii) SCUBA exploration and later robotsampling in oceanic depths. The development of synthetic chemistry soon became necessary to provide alternative sources for rare organisms, but also to find better analogues (more active, less toxic, more soluble, better tolerated, easier and cheaper to synthesize...). 
A new biotechnological turn has recently been taken with the high capacity screening of vast libraries of synthetic analogues, with the development of biomimetic chemistry and with the use of genetic tools that allow bacterial carriers like Escherichia coli to produce a compound of interest after insertion of the set of genes sampled from the original biological source.

Marine molecular sciences have now come of age, but the exploration of novel natural products and of new biological activities must go on, especially in relation to marine microbes and to planktonic organisms.

Many (98 to $99 \%$ ) of the known marine bacteria and archaea still cannot be cultivated using the existing apparatus and growth media. Knowing that invertebrates such as sponges, ascidians, bryozoans act as biological incubators of specific bacterial strains, isolated in specialized cells called bacteriocytes or allowed to interact synergistically, there is a world of possibilities opened for us to investigate. After all, we only discovered recently that bacteria have a form of social life within biofilms, have developed a chemical language that allows their populations to be regulated, have an instrumental role in carbon and nitrogen cycling in holobiont photosystems such as corals, lichens etc. and in biogeochemical cycles at large [28].

Indeed we still have no idea of the importance bacteria have in the production or in the modulation of the genetic expression of key metabolites hitherto attributed to their host organisms, except through limited attempts to cultivate the latter axenically. Using a systems biology approach, stress transcriptomics studies will undoubtedly help us achieve better control of the production of key enzymes or key defense metabolites from host organisms, as well as evaluate the optimal conditions for the maintenance of a rich and diverse microflora using metagenomics.

The purpose here is not to list the bioactive molecules that have been discovered, elucidated, synthesized, nor to detail their bioactivities. Excellent reviews are regularly updated on the subject, presented by natural products chemists and pharmacologists. Interactive databases include standard spectral information, and sophisticated algorithms are available as aids for structure elucidation and as dereplication tools. Two recent and outstanding information sources are [31] and its updated version [33], and [34].

Well over 20,000 secondary metabolites have been screened so far, but only a handful is now legally approved for use as prescription drugs or treatments by the Food and Drugs Administration (FDA) and by the European Union since 1974 (see [36] and updates thereof in: http:// marinepharmacology.midwestern.edu/clinPipeline.htm). Successful molecules and analogues have to undergo in-vitro, in-vivo (preclinical) tests and finally several rounds of clinical trials on patients, a process known as the clinical pipeline. On average, each development costs about one billion dollars over a period of ten years, and there is great pressure to discover and develop novel molecules, especially against various types of cancer, antibiotics against resistant strains, and molecules for the treatment of neurodegenerative diseases. With energy, food and housing, pharmaceuticals represent a primary resource in modern economy. 


\subsection{High-tech}

Siliceous skeletons are transformed from dissolved silicic acid into very elaborate structures by diatoms and radiolarians. They not only serve a structural function: they allow sunlight to penetrate for photosynthesis, and there is evidence that harmful radiations are filtered out somewhat like our sunglasses, using embedded mycosporine-like amino acids as DNAprotective sunscreens [37]. Enzymes that are capable of creating optical-grade glass at room temperature are under investigation. Siliceous sponges produce glass spicules which are endowed with mechanical and optical properties unknown to manufacturers, and novel enzymatic approaches using sponge silicateins are investigated for nanotechnological and biomedical applications [38].

Shells of bivalve and gastropod mollusks, other than their interest as decorative items, often present amazing properties. Oyster nacre has osteo-inductive properties that could be used in bone tissue regeneration [39]. Abalone shells offer strong mechanical resistance to impact, thanks to its dual organic-limestone microscopically layered structure, inspiring novel bulletproof materials. Skeletons that are produced by sessile marine invertebrates are a great source of inspiration: some have tremendous flexibility and resilience to currents, like the horny skeleton of some gorgonians [40]; coral skeletons are used as temporary bone implants [41], coralline algae cements have amazing particle-aggregating properties, etc. Novel adhesives with high tensile strength are inspired from byssus filaments of mussels. Crab shells can provide a sustainable source of chitin and chitosan, which are used in a variety of medical and laboratory applications [42].

Bacteria can be modified genetically to produce pure toxins, antibiotics, polymers and enzymes with high added value. Cultivatable bacterial strains offer exciting possibilities for the transformation or the recycling of a wide range of industrial materials and agricultural waste products. Experimental biofilms can produce weak currents with potential applications in sewage treatment plants.

\section{Management of biodiversity and chemodiversity}

Global anthropic influence is articulated on the exploitation of natural resources and the generation of wastes. Here are a few examples of man's use of biogenic resources, mineral and organic, fossil or live.

\subsection{Urban development}

The use of biogenic sand and limestone concretions usually involves no direct utilization of live organisms. Indirectly, the overexploitation of sand beds from littoral zones represents a double problem: the eradication of the epibenthic wildlife and with time, erosive changes in coastal profiling. Sand particles offer extended surfaces that are essential for microorganisms to recycle decaying organic debris and avoid the accumulation of toxic nitrites and nitrates. The exploitation of sands from coral reef atolls, together with the silting of coastal bays and leeward zones due to mining activities spillage are major sources of biodiversity loss. 


\subsection{Energy}

The use of fossil hydrocarbons extracted from the Mexican Gulf, the Northern Sea etc., as energy sources results in the volatilization of carbon in the atmosphere at rates up to one million times faster than it took for phytoplankton to fix and sequester it into sediments as oil beds [25]. Atmospheric accumulation of carbon dioxide, methane, sulphur dioxide and other combustion products, create an artificial greenhouse effect after only 150 years of exploitation (now largely marine), adding to natural volcanic activity. Global warming and ocean acidification are both regarded as major potential sources of marine biodiversity destruction [43].

Biodiversity loss seems to be the inevitable toll to pay for a global economic expansion relying heavily on the use of biogenic productions. When such exploitations occur at the vicinity of marine biodiversity hotspots (coral reefs for example), which concentrate much of the useful and yet unexplored chemical diversity [26], we run into the risk of losing a lot of useful model molecules and end up with a hostile, impoverished microbial world instead [44].

\subsection{Drugs and ethics}

Drugs are basically dangerous when ingested, injected or applied externally without caution, and man has always made the difference between edibles and toxic or venomous substances, or foodstuff corrupted with microbes. Medicine men and witch doctors knew empirically how to use fresh plants and how to blend them to cure fevers, wounds and other ailments, and this knowledge was passed on exclusively to younger initiates who henceforth gained access to special social status and were highly respected. Today, bioactive substances are patented and the administration of prescription drugs is tightly controlled. As mentioned earlier, the drug industry is one of the very few primary sources of revenue in any country's economy. Exploration of novel sources of natural products in biodiversity hotspots, terrestrial or marine, is a touchy subject. Intellectual property should be shared in the benefit of local populations, eventually leading to their active participation in the cultivation and the preparation and purification of extracts, on a non-destructive and sustainable basis. This is especially true for developments that were guided by ethnopharmacological surveys, i.e. the participation of local medicine men. Marine bioexploration, however, is almost exclusively based on systematic screening of marine plants, invertebrates and microbes, but it should be subjected to territorial laws like the exploitation of forests, fishing zones or mines in a participative manner. More specifically, endangered or rare species should be identified, and sampling procedures be conducted in accordance with internationally established practices [45].

\subsection{Food}

We have purposely ignored aspects directly related to food, i.e. the exploitation of marine resources through fisheries and the farming of selected fish and seafood species. The sustainable management of natural stocks and the influence of global warming on natural biodiversity profiles is a major concern in the 21st century. Human population has almost trebled since WWII, and Homo sapiens dominates terrestrial vertebrate biomass (half a billion tons) to the point that meat protein supplies will be scarcer. After being a hunter-gatherer, man has become 
a farmer and he is now turning towards artificially produced biomass that has food value, grown from cultured transgenic cells. What next?

The management of natural sanctuaries that are subjected to minimal interference, with sizes large enough to have a self-regenerating biodiversity (and chemodiversity), and tightly controlled environmental connectivity, should be encouraged.

\section{Pollution}

Man does not always recycle the wastes he generates, far from it.

\subsection{Urban developments}

There are two major issues to consider: one is structural and the other is biological.

Structural issues are linked to urban planning and the exploitation of natural zones, and to the fate of obsolete constructions and roads that cannot be recycled back into their original components - the net result being a shift from marine destructive exploitation (sand) to terrestrial pollution (discharges). Wherever possible, alternatives to marine sand must be sought for housing, to save the resource and perhaps use recycled wood-based products and recyclable organic polymers.

Biological issues emerge from the disposal of untreated sewage, of fertilizers, pesticides, drugs, household maintenance products and industrial by-products. Regarding sewage, local enrichment of waterways and coastal areas, especially in nitrogen and phosphorus, causes toxic phytoplankton blooms, and unwanted algal proliferations that tend to displace indigenous species. This is especially true for complex oligotrophic ecosystems in which nutrients are efficiently used and recycled. In coral reefs, bacterial charges are introduced directly or via invasive algal proliferations [46], favoring the dominance of pathogenic strains and reducing the natural microbial diversity [47], [43].

\subsection{Energy}

Regarding energy-related matters, losses due extraction and transport (ship degassing, black tides due to wreckages etc.) are the cause of episodic but massive biodiversity destructions in littoral zones, by asphyxiating wildlife and by destroying skin protection barrier. The exploitation of light hydrocarbons from shales is currently the subject of intense debates, especially regarding its consequences on water quality [48]. The exploitation of gas hydrates represents an attractive energy source, but its exploitation may prove difficult to control, and its release from the thawing of the permafrost due to global warming difficult to evaluate [49].

\subsection{Synthetic drugs, artificial molecules, genetically modified organisms}

The modern world is global and highly competitive. Being positioned on an economic niche requires achieving results and outcompeting contenders, no matter what the human and 
environmental consequences are in the long term. Thousands of synthetic molecules - insecticides, herbicides, pharmaceuticals, conditioning agents, food additives, fuel additives, lubricants, household products, plasticizers, flame retardants, to name a few- have been synthesized without sufficiently questioning their degradability and speciation once released in the environment or abandoned, or offering detoxication, storage/disposal or reusability solutions. Highly reactive pesticides can cause immediate non-specific destruction to useful pollinizing insects and kill birds, others may insidiously find their way up the food chains and eventually poison top predators - this is the case of highly stable and carcinogenic biphenyl organohalogens. Chronic allergies and cancers are documented through the regular exposure to more and more food additives and household products. Specific information can be obtained from associative websites.

If there are no simple replacement solutions in most cases, perhaps revisiting global commercial practices, promoting local products and values and favoring educational resources (preferably non-virtual) centered on the practical resolution of local issues, will help establish respectable ethics and restore some dignity.

\section{Rearranging clouds in times of global changes?}

We are in the midst of the worldwide race for economic development and global trading, with their extraordinary demands on natural resources and their inevitable environmental issues. While striving to maintain social equilibrium at the same time, why not take a pause and ask ourselves not only where we are going, but simply how we are going? How can we know how we are going?

The prestigious graduate schools, the most popular jobs in western and industrialized countries are linked with the world of finance. Get closer to the sources, react faster and make the optimal decision before anyone else does. Sophisticated algorithms are being developed by highly paid experts to better analyze tendencies and make predictions, from a phenomenal amount of cross-analyzed sources of diverse natures. Lists of potential customer's addresses are sold, exchanged, stolen. Cloud metadata and then triturated and sent back to the average human as a continuous shower of commercial ads, enticing e-mails, spurious spams, in the hope of creating habits and develop ruinous compulsions? Along the same line, potential crime scenes can now be anticipated with amazing probability rates by the Californian police, thanks to the work of metadata experts.

Global changes are on the way, noticeable since the70's with the accelerating atmospheric enrichment of greenhouse gases. Science and technology have made extraordinary progress at the same time, but mostly dedicated to the continuing exploration of the unknown "before it is too late", and too little to addressing pressing environmental issues with the huge losses in biodiversity that are envisioned by many in the next decades.Cutting edge medical research has been fuelled by the need to face new challenges, in cancerology, in microbial pathologies, in immune or rare diseases. Highly efficient molecular investigations from gene mining to the knowledge and control of their expression repertoire, whole organism approaches, continuously updated cutting-edge equipment - are being applied to exploratory science and projec- 
tions into future scenarios. Huge supercomputers are piling up petabytes of data waiting for bioinformatics advances to organize and make sense of them, and in the quest of the reassuring "big picture" that will once for all settle intellectual disputes, quench the thirst for the unknown, and with the hope that, ultimately, some biogeoclimatic equilibrium will be automatically restored no matter what.

How most of us are doing depends not on fluctuations of the stock market, nor on the price of the latest hi-tech widget, nor on whether the next crime scene is going to take place in our neighborhood, but on how fast our natural environment is going to degrade. Why not redirect our priorities and efforts to better understand how our biosphere functions, how species interact, how biomes are connected, how biodiversity is maintained in marine and terrestrial environments?

We generate enormous amounts of data of genomic, chemical, taxonomic, pharmacological, biotechnological value that can be better analyzed.

Dealing with environmental issues, the first question is the preparation of environmental databases, our "clouds"- the crucial initial step. What "standard" environment will be suitable to monitor long-term changes? Climatic changes being global (temperature rises, ocean acidification etc.) and heavily influenced by human activities, one can only define a study zone as representative of a "standard environment" if it is not locally impacted by direct human interference (habitat destruction, introduction of alien species, chemical and microbiological pollution...). Fluctuations in parameters of interest must not be subjected to amplitudes higher than those of experimental (read: impacted) study zones, and sampling must be large and statistically significant for the "standard" database to be robust and reliable enough to evidence mild to severe changes in same parameters from experimental study zones. This applies not only to physico-chemical parameters, but also to genomics, transcriptomics or metabolomics metadata (e.g. microbial metagenomics). We need markers within variables, sentinel species within populations, signatures within metabolomes, early responses within complete transcriptomic repertoires, and so on. Some advocate the use of artificial biological systems, a synthetic biology approach, to standardize sampling procedures. Whole-area imaging can make use of drones that interfere very little with wildlife and biological processes. Coupled with appropriate mathematical tools, automation can be achieved and time-dependent evolution of e.g. natural processes can be predicted.

Thus, whether comparing experimental vs. control areas, or monitoring the evolution of the same locality in time, or analyzing connectivity, enormous amounts of data are being generated that need trimming, arranging, cross-checking to be usefully interpreted. And beyond assessing the evolution of a situation, we need to predict, for example, the degradation in the biodiversity of impacted areas if no corrective action is taken. Neural networks that are "educated" by so-called genetic algorithms are currently very successfully exploited, in economics, in business, and in forensic science, to open what is known as "windows of opportunities" (to seize, but also to take corrective measures). These were created by scientists to treat scientific questions in the first place! Beyond our biosphere's apparent complexity, what it contains are quantitatively and qualitatively finite entities, subjected to predictable dynamics. There is no reason not to apply these analytical methods to create ready-to-use tools legislation can rely upon to regulate the appropriation of natural resources: water, wood, sand, fossil fuel, minerals... and wildlife. In the same way as DNA tests are used to help solve 
criminal investigation by 'showing irrefutable evidence', molecular tools applied to systems biology in association with multi-scale imaging tools can be tailored by analysts to guide our choices towards a more responsible management of natural resources.

We need a generation of motivated young analysts to use their skills to extract the useful information and blow away the dust. The big pictures might then emerge at last, as the clouds dissipate, ready to inspire us with a better scenario. The following phrase by Salvatore Manga no [50] "Genetic Algorithms are good at taking large, potentially huge search spaces and navigating them, looking for optimal combinations of things, solutions you might not otherwise find in a lifetime" makes needle-in-the haystack problems tractable. After all, we live on a planet with finite resources, our only problem now is to make them last for the benefit of the generations to come. If machines can learn, hopefully humans can too....

\section{Acknowledgements}

I wish to thank Catherine Boyen of UMR 7139 at Station Biologique de Roscoff for financial assistance, and I wish to dedicate this work to John Saville Waid (1927-2012), an internationally known Professor and specialist of soil microbiology, whose friendliness and contagious smile will always be remembered by those who knew him.

\section{Author details}

Stéphane La Barre ${ }^{1,2}$

1 Université Pierre et Marie Curie-Paris 6, UMR 7139 Végétaux marins et Biomolécules, Station Biologique F-29680, Roscoff, France

2 CNRS, UMR 7139 Végétaux marins et Biomolécules, Station Biologique F-29680, Roscoff, France

\section{References}

[1] Valley JW, Peck WH, King EM, Wilde SA. A cool early earth. Geology 2002;30: 351-354.

[2] Miller SL A production of amino acids under possible primitive earth conditions. Science 1953;117: 528-529.

[3] Parker ET, Henderson JC, Dworkin JP, Glavin DP, Callahan M, Aubrey A, Lazcano A, Bada JL. Primordial synthesis of amines and amino acids in a 1958 Miller $\mathrm{H}_{2} \mathrm{~S}$-rich spark discharge experiment. PNAS 2011;108(14): 5526-5531. 
[4] Oró J, Kamat SS. Amino-acid synthesis from hydrogen cyanide under possible primitive earth conditions. Nature 1961;190 (4774): 442-3. Bibcode:1961 Natur.190.442O. doi:10.1038/190442a0

[5] Ritson D, Sutherland JD. Prebiotic synthesis of simple sugars by photoredox systems chemistry. Nature Chemistry 2012;4: 895-899.

[6] Shapiro R. Prebiotic ribose synthesis: a critical analysis. Origins of Life and Evolution of the Biosphere 1988;18: 71-85.

[7] Huber C, Wächterhäuser G. $\alpha$-hydroxy and $\alpha$-amino acids under possible Hadean, volcanic origin-of-life conditions. Science 2010;314: 630-632.

[8] Raulin F, Coll P, Navarro-González R. Prebiotic Chemistry: Laboratory Experiments and Planetary Observation. In: Gargaud M. et al. (eds.) Lectures in Astrobiology. Berlin, Heidelberg: Springer-Verlag; 2005;1: p449-471. ISBN: 978-3-540-22315-3

[9] Gilbert W. The RNA world. Nature 1986;319: 618.

[10] Ferris JP, Hill AR, Liu R, Orgel E. Synthesis of long prebiotic oligomers on mineral surfaces. Nature 1996;381, 59-61.

[11] Eschenmoser A. Chemical etiology of nucleic acid structure. Science 1999;284, 21182124.

[12] Maurel C, Haenni M-L. The RNA World: Hypotheses, Facts and Experimental Results. In: Gargaud M. et al. (eds.) Lectures in Astrobiology. Berlin, Heidelberg: Springer-Verlag; 2005;1: p571-594. ISBN: 978-3-540-22315-3.

[13] Meli M, Vergne J, Maurel M-C (2003). In vitro selection of adenine-dependent hairpin ribozymes. J. Biol. Chem., 2003;278(11): 9835-9842.

[14] Bada JL, Lazcano L. Some like it hot, but not the first biomolecules. Science 2002;296: 1982-1983.

[15] Balucani N. Elementary reactions and their role in gas-phase prebiotic chemistry. International Journal Of Molecular Sciences, 2009;10: 2304-2335; doi: 10.3390/ ijms10052304.

[16] Commeyras A, Boiteau L, Vandenabeele-Trambouze O, Selsis F. Peptide emergence, evolution and selection on the primitive earth. I. Convergent Formation of N -Carbamoyl Amino Acids Rather than Free $\alpha$-Amino Acids?. In: Gargaud M. et al. (eds.) Lectures in Astrobiology. Berlin, Heidelberg: Springer-Verlag; 2005;1: p517-545. ISBN: 978-3-540-22315-3.

[17] Chrysostomou A, Ménard F, Gledhill TM, Clark S, Hough JH, McCall A, Tamura M. Circular Polarization in Star Formation Regions: Implications for Biomolecular Homochirality. Science 1998;281(5377): 672. 
[18] Cronin J, Reisse J. Chirality and the Origin of Homochirality. In: Gargaud M. et al. (eds.) Lectures in Astrobiology. Berlin, Heidelberg: Springer-Verlag; 2005;1, p473-515. ISBN: 978-3-540-22315-3

[19] Ourisson G, Nakatani Y. A Rational Approach to the Origin of Life: from Amphiphilic Molecules to Protocells. Some Plausible Solutions and Some Real Problems. In: Gargaud M. et al. (eds.) Lectures in Astrobiology. Berlin, Heidelberg: Springer-Verlag; 2005;1: p429-448. ISBN: 978-3-540-22315-3

[20] Commeyras A, Boiteau L, Vandenabeele-Trambouze O, Selsis F. Peptide Emergence, Evolution and Selection on the Primitive Earth. II - The Primary Pump Scenario. In: Gargaud M. et al. (eds.) Lectures in Astrobiology. Berlin, Heidelberg: Springer-Verlag; 2005;1: p547-569. ISBN: 978-3-540-22315-3

[21] Szostak JW, Bartel DP, Luisi PL. Synthesizing life. Nature 2001;409: 387-390.

[22] Pinti M. The Origin and Evolution of the Oceans. In: Lectures in Astrobiology, vol. 1, 83-112, M. Gargaud et al. (Ed.), Springer-Verlag Berlin Heidelberg 2005 ISBN: 978-3-540-22315-3

[23] Benton MJ. When Life Nearly Died: the Greatest Mass Extinction of all Time. London: Thames \& Hudson 2005, 336 pages. ISBN-13: 978-0500285732.

[24] Knoll AH, Bambach RK, Canfield DE, Grotzinger JP. Comparative Earth history and Late Permian mass extinction. Science 1996;273(5274): 452-457.

[25] Falkowski P. Tenth Annual Roger Revelle Commemorative Lecture: The once and future ocean. Oceanography 2009;22(2): 246-251, doi:10.5670/oceang.2009.57.

[26] La Barre S. Novel tools for the evaluation of the health status of coral reefs and for the prediction of their biodiversity in the face of climatic changes. In: Zambianchi E. (ed.) Topics in Oceanography. Rijeka: InTech; 2013. p127-155.

[27] Dill KA, Truskett TM, Vlachy V, Hribar-Lee B. Modelling water, the hydrophobic effect and ion solvation. Annu. Rev. Biophys. Biomol. Struct. 2005;34: 173-99 doi: 10.1146/annurev.biophys.34.040204.144517

[28] Falkowski, PG, Fenchel; T, Delong, EF. The microbial engines that drive earth's biogeochemical cycles. Science 2008;320: 1034-1039.

[29] Waid JS. Does soil biodiversity depend upon metabiotic activity and influences? Applied Soil Ecology 1999;13: 151-158.

[30] Kornprobst J-M. Encyclopedia of Marine Natural Products. Weinheim: Wiley-Blackwell Verlag GmbH \& Co. KGaA; 2010. ISBN; 978-3-527-32703-4.

[31] Cani PD, Delzenne NM, Amar J, Burcelin R. Role of gut microflora in the development of obsesity and insulin resistance following high-fat feeding. Pathologie Biologie 2008;56: 305-309, doi: 10.1016/j.patbio.2007.09.008 
[32] Hay ME, Fenical W. Marine plan-herbivore interactions: the ecology of chemical defense. Annual Review of Ecology and Systematics, 1988;19: 111-145.

[33] Kornprobst J-M. Encyclopedia of Marine Natural Products. Weinheim: Wiley-Blackwell Verlag GmbH \& Co. KGaA; in press; ISBN; 978-3-527-33429-2 (printed version) and ISBN; 978-3-527-33585-5 (electronic version).

[34] Fattorusso E; Gerwick WH, Tagliatela-Scafati O (editors). Handbook of Marine Natural products, 2011, 2 volumes, 691 Figures, 45 Tables, Springer Science, Print and electronic bundle under ISBN 978-90-481-3856-2, DOI: 10.1007/978-90-481-3834-0

[35] Lopanik, N.B. (2013) Chemical defensive symbioses in the marine environment. Functional Ecology, in press. doi: 10.1111/1365-2435.12160

[36] Mayer A, Glaser KB, Cuevas C., Jacobs, RS; Kem W, Little RD, McIntosh JM, Newman DJ, Potts BC, Shuster DE. The odyssey of marine pharmaceuticals: a current pipeline perspective. Trends in Pharmacological Sciences 2010;31: 255-265. doi: 10.1016/j.tips.2010.02.005

[37] Ingalls AE, Whitehead K, Bridoux MC. Tinted windows: the presence of the UV absorbing compounds called mycosporine-like amino acids embedded in the frustules of marine diatoms. Geochimica et Cosmochimica Acta 2010;74: 104-115. doi:10.1016/ j.gca.2009.09.012.

[38] Müller EG, Wang X, Cui F-Z, Jochum KP, Tremel W, Bill J, Schröder HC, Natalio F, Schloßmacher, Wiens M. Sponge spicules as blueprints for the biofabrication of inorganic-organic composites and biomaterials. Appl Microbiol Biotechnol 2009;83: 397413.

[39] Rousseau M. Nacre, a natural biomaterial. In: Pignatello R. (ed.) Biomaterials Applications for Nanomedicine. Rijeka: InTech, 2011, p661-664.

[40] Jeyasuria P, Lewis JC. Mechanical properties of the axial skeleton in gorgonians. Coral Reefs 1987;5, 213-219.

[41] Demers C, Hamdy CR, Corsi K, Chellat F, Tabrizian M, Yahia L. Natural coral exoskeleton as a bone graft substitute: a review. Bio-Medical Materials and Engineering, 2002;12(1): 15-35.

[42] Hirano S. Chitin biotechnology applications. Biotechnology Annual Review, 1996;2: 237-258.

[43] La Barre S. Coral reef biodiversity in the face of climatic changes. In: Grillo, O. (ed.) Biodiversity Loss in a Changing Planet. Rijeka: InTech; 2011. p77-112.

[44] Rohwer F. Coral reefs in the microbial seas. USA: Plaid Press; 2010, 201 pages, ISBN: 978-0-9827012-0-1.

[45] Crag GM, Katz F, Newman DJ, Rosenthal J. Legal and ethical issues involving marine biodiversity and development. In: Fattorusso E, Gerwick WH, Tagliatela-Scafati 
O (editors). Handbook of Marine Natural Products. Springer Science 2011;2: p1315-1342. Print and electronic bundle under ISBN 978-90-481-3856-2, DOI: 10.1007/978-90-481-3834-0.

[46] Hay ME, Rasher DB. Coral reefs in crisis: reversing the biotic death spiral. F1000 Biology Reports 2010;2(71) (doi:10.3410/B2-71).

[47] Mouchka ME, Hewson I, Harvell D. Coral-associated bacterial assemblages: current knowledge and the potential for climate-driven impacts. Integrative and Comparative Biology, 2010;50(4): 662-674. ISSN: 1540-7063.

[48] Al T, Butler K, Cunjak R, MacQuarrie K. Opinion: Potential Impact of Shale Gas Exploitation on Water Resources. 2012. University of New Brunswick Report.

[49] Kvenvolden KA. Gas hydrates - geological perspective and global change. Reviews of Geophysics 1993;31(2): 173-187.

[50] Mangano S. Computer Design (Powerpoint presentation, 1995) https://www.cs.drexel.edu/ spiros/teaching/SE320/slides/ga.pdf. 